\title{
Cuantificación de bacterias relacionadas con la caries dental en saliva de adultos y adultos mayores
}

\author{
Quantification of caries-associated bacteria from saliva \\ of adults and older adults
}

\author{
Giacaman RA ${ }^{1}$, Muñoz-Sandoval $C^{1}$, Bravo González E ${ }^{1}$, Farfán-Cerda $\mathrm{P}^{1}$
}

\begin{abstract}
RESUMEN
Introducción: Se considera que Streptococcus mutans (S. mutans) y Lactobacillus spp. se asocian con la caries. Otras especies del biofilm oral, como Streptococcus sanguinis (S. sanguinis) han sido sindicadas como protectoras, pero principalmente en niños. Existe escasa evidencia sobre el nivel de estas bacterias en adultos mayores. Objetivo: Determinar si existen diferencias en los recuentos microbianos de tres especies relacionadas con la caries en pacientes adultos y adultos mayores. Materiales y Métodos: Una muestra de pacientes por conveniencia compuesta de 63 pacientes (18 a 79 años) proporcionó saliva estimulada con la que se sembraron placas de agar MSB, MM10 SB y Agar Rogosa para el cultivo de $S$. mutans, $S$. sanguinis y Lactobacillus spp., respectivamente. Los recuentos bacterianos fueron expresados como UFC/mL. Resultados: Los recuentos de S. mutans y Lactobacillus spp. no mostraron variaciones relacionadas con la edad $(p>0.05)$. Los adultos mostraron mayores recuentos de $S$. sanguinis que los adultos mayores, $3.7 \times 10^{5} \pm 3.8 \times 10^{5} \mathrm{UFC} / \mathrm{mL}$ y $5.9 \times 10^{4} \pm 9.4 \times 10^{4} \mathrm{UFC} / \mathrm{mL}$, respectivamente $(p<0.05)$. Conclusiones: La edad no parece afectar los niveles de especies tradicionalmente consideradas como cariogénicas. Estos resultados sugieren que la edad puede relacionarse con los patrones de colonización de S. sanguinis en el biofilm oral.
\end{abstract}

Rev. Clin. Periodoncia Implantol. Rehabil. Oral Vol. 6(2); 71-74, 2013.

Palabras clave: S. mutans, S. sanguinis, Lactobacillus spp., adultos mayores, caries, biofilm oral.

\begin{abstract}
Introduction: Streptococcus mutans (S. mutans) and Lactobacillus spp. have been traditionally associated with caries, regardless of the subject's age. Other oral biofilm species have been linked as caries protective, including Streptococcus sanguinis (S. sanguinis), but mainly in children. Scarce evidence exists on the levels of these bacteria in older adults. Aim: To determine whether there are differences in the microbial counts of three caries-associated bacterial species in adults and older adults. Methodology: A convenience sample of sixty three patients, aged 18 to 79 years, participated in the study. Stimulated saliva samples were obtained and in MSB, MM10 and Rogosa agar plates for the culture of S. mutans, S. sanguinis and Lactobacillus spp., respectively. Bacterial counts were obtained by microscopic observation (10x) of the colonies and expressed in CFU/mL. Results: Bacterial counts of $S$. mutans and Lactobacillus spp. did not reveal age-related differences ( $p>0.05)$. Adults showed higher $S$. sanguinis counts than older adults with $3.7 \times 10^{5} \pm 3.8 \times 10^{5} \mathrm{CFU} / \mathrm{mL}$ and $5.9 \times 10^{4} \pm 9.4 \times 10^{4} \mathrm{CFU} / \mathrm{mL}$, respectively ( $p<0.05$ ). Conclusions: Age does not seem to affect the levels of bacterial species traditionally associated with caries. The results suggest that age may be related to colonization patterns of $S$. sanguinis in the oral biofilm.
\end{abstract}

Rev. Clin. Periodoncia Implantol. Rehabil. Oral Vol. 6(2); 71-74, 2013.

Key words: S. mutans, S. sanguinis, Lactobacillus spp., older adults, caries, oral biofilm.

\section{INTRODUCCIÓN}

La caries continúa siendo un problema altamente prevalente en todo el mundo(1). Particular interés reviste la caries radicular en el adulto mayor, dado el explosivo aumento demográfico de la población ${ }^{(2)}$. El envejecimiento conlleva numerosos cambios, los que repercuten en las condiciones medioambientales orales. Durante el desarrollo de la dentición, el número y tipo de microorganismos orales cambian acorde avanza la edad, particularmente en edades tempranas ${ }^{(3)}$. La cavidad oral es colonizada por diferentes microbiotas según la topografía en que se encuentren. Se han descrito más de 700 especies bacterianas que pueblan cada uno de estos nichos ecológicos ${ }^{(4)}$.

En la concepción actual de la etiopatogenia de la caries, cambios medioambientales orales generan predominancia de algunas especies con potencial acidogénico, necesario para la generación de lesiones de caries $^{(4)}$. En ese estado de desequilibrio, la flora oral muestra composiciones diferentes a las que se observan en salud. Así, por ejemplo, las especies tradicionalmente asociadas con lesiones de caries incluyen a Streptococcus mutans (S. mutans), S. sobrinus,
Actinomyces spp. y Lactobacillus spp. ${ }^{(5)}$. Se ha reportado que dichas especies cariogénicas se mantendrían constantes independientemente de la edad de los sujetos ${ }^{(6-8)}$, aunque la evidencia al respecto es limitada y escasa. Por años se consideró a S. mutans como el principal agente etiológico de caries dental(9). Su rol patogénico implicaba la iniciación de la desmineralización del esmalte en la caries coronal y de la superficie de la raíz en las caries radiculares ${ }^{(10)}$. Su presencia es ubicua en la boca, tanto en placa como en saliva de adultos y adultos mayores ${ }^{(11)}$. Pese a su marcado potencial cariogénico, su asociación con la caries, en términos numéricos no es clara ${ }^{(12)}$ y se mantiene como un tema controversial. Por otro lado, Streptococcus sanguinis (S. sanguinis) es uno de los colonizadores tempranos del biofilm oral. Este microorganismo constituye aproximadamente el $15 \%$ de la microflora oral, lo que se traduce en altos recuentos de $S$. sanguinis en saliva(13). En un papel antagónico con $S$. mutans, $S$. sanguinis se ha asociado con sitios libres de la enfermedad. Las investigaciones revelan que $S$. sanguinis podría competir con $S$. mutans por la colonización de superficies dentarias desde el momento en que se produce la erupción de los dientes ${ }^{(14,15)}$. En niños, se ha descrito una asociación directa entre la colonización por $S$. sanguinis y

1. Unidad de Cariología. Departamento de Rehabilitación Buco-Máxilofacial, Universidad de Talca. Talca, Chile.

Correspondencia autor: Rodrigo A. Giacaman. giacaman@utalca.cl. Trabajo recibido el 08/11/2012. Aprobado para su publicación el 01/03/2013. 
la cantidad de dientes libres de caries e inversa en aquellos dientes que presentan las lesiones ${ }^{(16)}$. Los mecanismos de antagonismo bacteriano entre estas dos especies incluyen la formación de mutacinas por parte de S. mutans y la de peróxido de hidrógeno por parte de $S$. sanguinis ${ }^{(15)}$. A pesar que esta asociación ha sido descrita en términos de la colonización bacteriana global de la boca en niños y adolescentes ${ }^{(14,15,17,18)}$, existe escasa información respecto a la relación $S$. mutans y $S$. sanguinis en adultos y adultos mayores.

Otra especie relacionada con la etiología y patogénesis de la caries son especies de Lactobacillus spp. Dichos microorganismos se asocian mayormente con la colonización de zonas retentivas creadas por las lesiones en las que quedan atrapados físicamente, aumentando en número durante la progresión y avance de la caries ${ }^{(19)}$. Además de la presencia de especies típicamente consideradas como cariogénicas para caries radicular, existen otras bacterias que se sumarían al proceso en adultos mayores, constituyéndose así un biofilm de alta complejidad como lo revelan estudios recientes ${ }^{(20)}$. Pese a ello, no hay claridad sobre el tema, el que resulta relevante para comprender de mejor manera la problemática particular que enfrentan los adultos mayores en términos de caries. De allí que el objetivo del presente estudio fue comparar los recuentos salivales de algunas especies del biofilm oral con un rol en la caries dental; S. mutans, S. sanguinis y Lactobacillus spp., en adultos y adultos mayores de especies tradicionalmente relacionadas con caries de las superficies de la raíz dentaria.

\section{MATERIALES Y MÉTODOS}

\section{Sujetos}

Se realizó un estudio de corte transversal con sujetos adultos y adultos mayores invitados a participar. Para ser incluidos en el estudio los sujetos debían tener entre 18 y 39 años para los adultos y 60 años o más para los adultos mayores. Se excluyeron de esta investigación los desdentados totales, fumadores, consumidores de alcohol, los que se encontraban o hayan recibido tratamiento de quimioterapia o radioterapia de cabeza y cuello, embarazadas y todos los sujetos que hubiesen recibido terapia con antibióticos durante los últimos 6 meses. Los participantes debían firmar un consentimiento informado aprobado por el Comité de Bioética de la Universidad de Talca, el que también aprobó el protocolo del estudio.

\section{Toma de Muestra Salival}

Cada paciente fue instruido en no comer, lavar o enjuagar sus dientes al menos una hora antes de la toma de muestra de saliva para el examen microbiológico. De ser necesario, sólo podían beber agua. Las muestras de saliva fueron recolectadas mediante flujo salival estimulado $(5 \mathrm{~mL})^{(21)}$. Las muestras fueron trasladadas refrigeradas al laboratorio $\left(4^{\circ} \mathrm{C}\right)$ para su posterior procesamiento microbiológico.

\section{Estudio Microbiológico}

La saliva fue homogeneizada en un agitador de tubos (Maxi Mix II tipo 37600 Mixer, Panel, EE.UU.) durante $30 \mathrm{seg}$. Luego, $100 \mu \mathrm{L}$ de la muestra fueron diluidos seriadamente en $900 \mu \mathrm{L}$ de $\mathrm{NaCl}$ al $0.9 \%$ ( $v / v)$ hasta la dilución 1: 1000. A partir de la dilución final, $50 \mu \mathrm{L}$ fueron sembrados sobre placas de agar Mitis-Salivarius-sacarosa-bacitracina (MSB) (BD, Sparks, EE.UU.)(22), altamente selectivo para S. mutans. Otros $50 \mu \mathrm{L}$ fueron transferidos a placas de agar del medio MM10 agar sangre sacarosa (MM10 SB agar), medio no selectivo diferencial para S. sanguinis ${ }^{(23)}$. Una última alícuota de $50 \mu \mathrm{L}$ de la muestra diluida sirvió para sembrar dos placas por cada participante del estudio de Agar Rogosa (Merck, Darmstadt, Alemania) (24), selectivo para Lactobacillus spp. Una vez sembradas, las placas fueron incubadas anaeróbicamente en jarras mediante la adición de un sobre de anaerobiosis comercial con indicadores (Anaeropack, Mitsubishi Gas, Chemical Company, Inc. Japón) a $37^{\circ} \mathrm{C}$ por $48 \mathrm{~h}$ para S. mutans y Lactobacillus spp. y $72 \mathrm{~h}$ para $S$. sanguinis. Transcurrido el tiempo de incubación se procedió al recuento de colonias. Las colonias de cada especie fueron identificadas fenotípicamente sobre las placas de Petri utilizando una lupa Spencer (10x). Los parámetros de identificación fenotípica utilizados para $S$. mutans incluyeron aquellas colonias adherentes, café grisáceas, con superficie rugosa, apariencia de vidrio esmerilado, de consistencia dura, y que no pueden ser disgregadas cuando se manipulan con un asa de platino ${ }^{(25)}$. Las colonias de $S$. sanguinis fueron identificadas como aquellas firmes, adherentes, de forma estrellada y que no pueden ser disgregadas ${ }^{(26,27)}$. Para la identificación de los Lactobacillus spp., se contaron las colonias que mostraran superficies convexas, lisas, circulares y con bordes regulares ${ }^{(24)}$. La cantidad total de colonias presentes en las placas de Petri se obtuvo mediante el recuento de las colonias individuales, ajustando por el factor de dilución utilizado para cada tipo de cultivo, obteniéndose así el número final de unidades formadoras de colonias por $\mathrm{mL}(\mathrm{UFC} / \mathrm{mL})$.

\section{Análisis Estadístico}

La variable dependiente del estudio; recuento de especies, fue comparada entre los grupos de sujetos adultos y adultos mayores. Se verificó la normalidad en la distribución de los datos mediante el test de Smirnov Kolmogorov, constatándose que la ausencia de normalidad, por lo que las comparaciones fueron realizadas mediante la prueba noparamétrica de U. de Mann-Whitney utilizando el programa SPSS v.14.0.

\section{RESULTADOS}

Un total de 63 sujetos participaron en este estudio de corte transversal (31 adultos; 15 hombres y 16 mujeres y 32 adultos mayores 16 hombres y 16 mujeres), seleccionados por conveniencia de entre los pacientes en atención en el Centro de Clínicas Odontológicas de la Universidad de Talca (Tabla 1). El promedio de edad de los pacientes adultos fue $27.46( \pm 3.75)$ años, mientras que el de adultos mayores fue de 65.68 ( \pm 6.12$)$ años.

Al análisis de los recuentos bacterianos, no se detectaron diferencias $(p>0.05)$ en las cantidades de $S$. mutans entre adultos y adultos mayores con recuentos de $2.3 \times 10^{5} \pm 3.3 \times 10^{5}$ y $2.4 \times 10^{5} \pm$ $2.8 \times 10^{5} \mathrm{UFC} / \mathrm{mL}$, respectivamente (Figura 1). En cambio, los recuentos de $S$. sanguinis fueron mayores $(p<0.05)$ en los adultos que los adultos mayores, con un promedio de $3.7 \times 10^{5} \pm 3.8 \times 10^{5} \mathrm{UFC} / \mathrm{mL}$ y $5.9 \times$ $10^{4} \pm 9.4 \times 10^{4} \mathrm{UFC} / \mathrm{mL}$, respectivamente (Figura 2). Respecto de las cantidades de Lactobacillus spp., no se lograron apreciar diferencias significativas $(p<0.05)$ entre los grupos en estudio con recuentos de $2.6 x$ $10^{6} \pm 3.7 \times 10^{6} \mathrm{UFC} / \mathrm{mL}$ y $2.4 \times 10^{6} \pm 3.3 \times 10^{6} \mathrm{UFC} / \mathrm{mL}$, respectivamente (Figura 3). Adicionalmente, se realizaron comparaciones por sexo entre los adultos y adultos mayores y al interior de cada uno de ellos, sin encontrar diferencias $(p>0.05)$.

Tabla 1. Distribución de pacientes por edad y género.

\begin{tabular}{|c|c|c|c|c|c|c|}
\hline Edad & $\begin{array}{c}\text { Masculino } \\
(\mathbf{n})\end{array}$ & $\%$ & $\begin{array}{c}\text { Femenino } \\
(\mathbf{n})\end{array}$ & $\%$ & Total & $\%$ \\
\hline $18-39$ & 15 & 23.8 & 16 & 25.4 & 31 & 49.2 \\
\hline$\geq 60$ & 16 & 25.4 & 16 & 25.4 & 32 & 50.8 \\
\hline Total & 31 & 49.2 & 32 & 50.8 & 63 & 100 \\
\hline
\end{tabular}

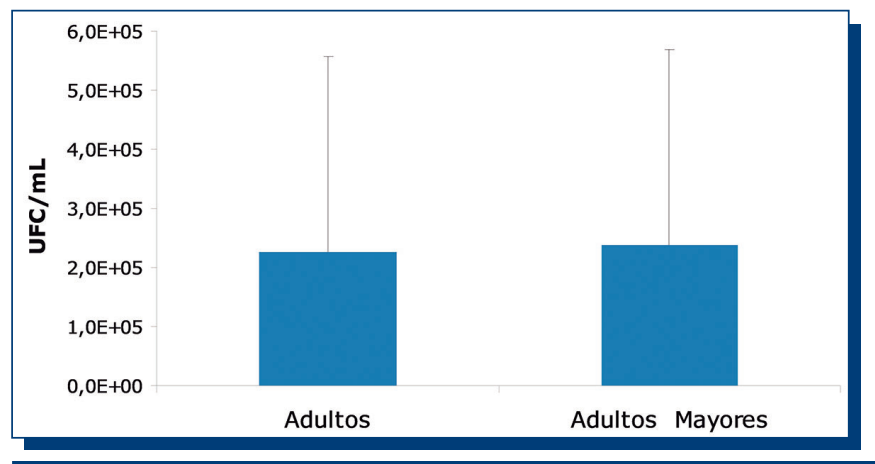

Figura 1. Recuento $S$. mutans de adultos y adultos mayores. Las barras indican el recuento promedio de S. mutans según grupo etario. Las barras de error indican la ds. 


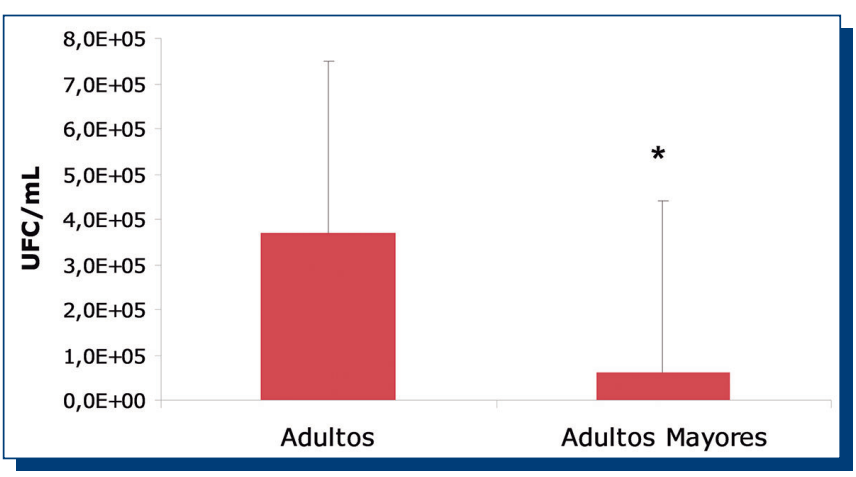

Figura 2. Recuento S. sanguinis de adultos y adultos mayores. Las barras indican el recuento promedio de $S$. sanguinis según grupo etario. Las barras de error indican la ds $\left({ }^{*}=p \leq 0.05\right)$.

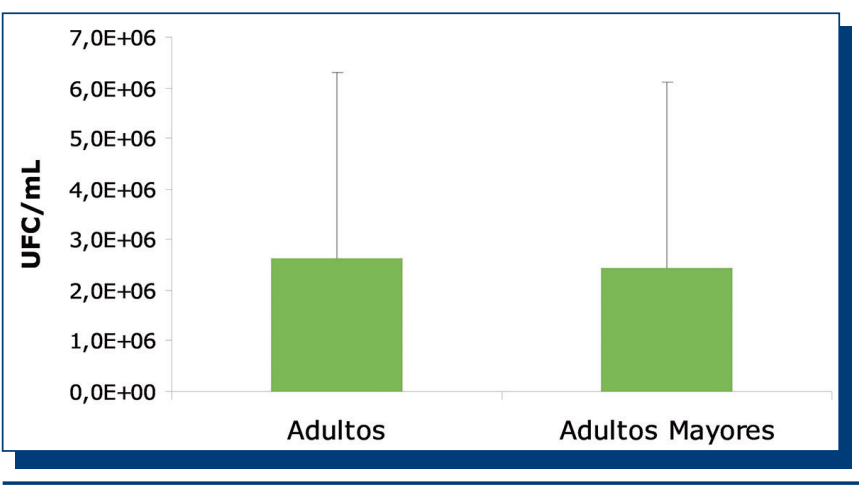

Figura 3. Recuento Lactobacillus spp. de adultos y adultos mayores. Las barras indican el recuento promedio de Lactobacillus spp. según grupo etario. Las barras de error indican la ds.

\section{DISCUSIÓN}

El biofilm oral es una consorcio bacteriano organizado y metabólicamente activo compuesto de cientos de especies bacterianas diferentes embebidas en una matriz de polisacáridos ${ }^{(4)}$. Es razonable especular que los cambios asociados a la edad afecten de cierta forma la ecología oral. Alteraciones en la saliva y en el flujo salival producto del consumo de fármacos, la secreción de anticuerpos, la exposición radicular ${ }^{(28)}$, son algunos de los múltiples factores que pueden condicionar un cambio en las condiciones locales orales que pueden afectar consecuentemente la composición del biofilm oral.

A otras edades del ciclo vital se han descrito cambios del biofilm oral. Los cambios relacionados con el crecimiento durante la pubertad y aquellos asociados a la erupción de las piezas dentarias ${ }^{(3)}$ crean situaciones que favorecen la proliferación de ciertas especies. En los adultos, se cree que la microflora se mantiene relativamente estable a menos que el ambiente se altere, como ocurre durante la enfermedad ${ }^{(5)}$. Sin embargo, existen pocos estudios sobre la microflora oral en individuos de avanzada edad(11,20). Los resultados de esta investigación sugieren que $S$. sanguinis fluctúa en sus niveles en relación a la edad. Así, adultos mayores muestran menores recuentos de este microorganismo que adultos más jóvenes. Por otro lado, otras especies consideradas tradicionalmente cariogénicas ${ }^{(5)}$, como $S$. mutans y los Lactobacillus spp., no parecen ser afectadas en cantidad entre ambos grupos etarios. Estos resultados son consistentes con investigaciones que no revelan diferencias etarias en los recuentos de $S$. mutans ${ }^{(11)}$. En la misma dirección, se ha reportado que incluso los recuentos de estos microorganismos supuestamente cariogénicos no se relacionan con tasas diferenciales de caries en una población adulta con alta experiencia de caries ${ }^{(12)}$. La relación de estas especies con la caries, más bien radicaría en una mayor expresión de factores de virulencia como los polisacáridos de adhesión secretados por ellos. Es importante destacar que las metodologías empleadas en este estudio para la identificación y recuento bacteriano son técnicas microbiológicas básicas ampliamente utilizadas en la literatura. Sin embargo, los desarrollos actuales de técnicas moleculares para la detección y recuento de especies podrían mejorar la precisión de estos hallazgos. Otro punto importante de destacar es que en este trabajo no se registró la historia de caries de los participantes debido a que el objetivo era analizar diferencias entre ambos grupos etarios. Pese a ello, futuros estudios deberían considerar la asociación de los niveles bacterianos con la experiencia de caries a fin de analizar una posible relación causal.

La evidencia disponible sobre la microflora oral asociada al proceso de caries en adultos mayores es escasa. Se ha descrito que además de las especies típicamente asociadas a la caries radicular como S. mutans, Actinomyces y Lactobacillus spp, otras especies se asocian fuertemente con superficies radiculares de adultos mayores ${ }^{(20)}$. Menos claro, sin embargo, el biofilm de adultos mayores en condiciones de salud no ha sido bien caracterizado. Por otro lado, se ha mostrado que los recuentos de $S$. mutans aumentan con la severidad de la enfermedad periodontal(29), la que también aumenta con la edad. Pese a que se consideraba tradicionalmente que $S$. mutans y Lactobacillus $s p p$. aumentaban directamente con la actividad de caries ${ }^{(30)}$, recientes investigaciones muestran que los niveles de Lactobacillus spp. en superficies radiculares con y sin caries no difieren significativamente entre $\mathrm{si}^{(31)}$. Dada la falta de correlato entre caries y los recuentos de $S$. mutans y Lactobacillus spp, en esta investigación los pacientes fueron reclutados sin considerar la presencia de caries.

En el caso de $S$. sanguinis, se constató un mayor recuento en grupos de adultos que en adultos mayores. Dado que $S$. sanguinis es un colonizador temprano del biofilm oral es esperable que se asocie más a superficies dentarias duras, por lo que en sujetos jóvenes que habitualmente poseen mayor número de dientes, podrían aislarse más microorganismos de esta especie que en adultos mayores que frecuentemente han perdido gran cantidad de dientes ${ }^{(32)}$.

Los hallazgos de la presente investigación que muestran proporciones diferenciales de $S$. sanguinis en adultos y adultos mayores resultan de alto interés en odontología. $S$. sanguinis parece interactuar con S. mutans y competir por la colonización del biofilm que se forma sobre los tejidos mineralizados dentarios ${ }^{(17)}$. Esta competencia tendría lugar desde la erupción de los dientes ${ }^{(18,33,34)}$. La bacteria $S$. sanguinis sería más efectiva en colonizar superficies libres de caries, mientras que S. mutans estaría más ligado a la colonización de dientes con lesiones activas $^{(16,35)}$. Pese a que esta asociación ha sido descrita en términos de la colonización bacteriana global de la boca en niños y adolescentes ${ }^{(17,36-38)}$, existe escasa información respecto a la relación de $S$. mutans y $S$. sanguinis en adultos ${ }^{(16)}$. Las complejas condiciones de cultivo de estos microorganismos, junto con una distribución no homogénea de ellos en la cavidad oral pueden influir en la carencia de estudios en poblaciones adultas $^{(39)}$.

Se ha especulado que el aumento relativo del número de $S$. mutans por sobre el de $S$. sanguinis podría servir como indicador de riesgo de caries ${ }^{(35,40,41)}$. La presencia de $S$. mutans por sí solo puede no ser el único indicador de riesgo ${ }^{(12)}$, como lo hemos reportado previamente. Estos hallazgos abren el camino para nuevas investigaciones tendientes a indagar sobre el rol de $S$. sanguinis en el proceso de caries de adultos y particularmente de adultos mayores. Las potenciales aplicaciones de estos resultados incluyen el desarrollo de exámenes microbiológicos diagnósticos basado en los recuentos de $S$. sanguinis para incorporarlos en la determinación de riesgo de caries. Aún así, estos hallazgos, sólo pueden ser considerados como datos preliminares y nuevas investigaciones se hacen necesarias para ahondar en el tema.

\section{RECONOCIMIENTOS}

Los autores agradecen la colaboración del Dr. Sebastián Torres en la siembra y recuento de las bacterias. Todos los autores han contribuido al estudio y han revisado la versión final del manuscrito. Los autores no declaran conflictos de interés. Este estudio fue financiado por el proyecto Fondecyt 11100005 a RAG.

\section{CONFLICTO DE INTERESES}

Los autores declaran no tener conflictos de interés. 


\section{REFERENCIAS BIBLIOGRÁFICAS}

1. Petersen $P$, Bourgeois D, Ogawa $H$, Estupinan-Day S, Ndiaye C. The global burden of oral diseases and risks to oral health. Bull World Health Organ, 2005; 83(9): 661-669.

2. Shrestha LB. Population aging in developing countries. Health Aff (Millwood), 2000;19(3): 204-212.

3. Socransky SS, Manganiello SD. The oral microbiota of man from birth to senility. J Periodontol, 1971; 42(8): 485-496.

4. Marsh PD. Microbiology of dental plaque biofilms and their role in oral health and caries. Dent Clin North Am, 2010; 54(3): 441-454

5 . Takahashi N, Nyvad B. The role of bacteria in the caries process: Ecological perspectives. J Dent Res, 2011; 90(3): 294-303.

6. Bowden GH, Ekstrand J, McNaughton B, Challacombe SJ. Association of selected bacteria with the lesions of root surface caries. Oral Microbiol Immunol, 1990; 5(6): 346-351.

7. Schüpbach P, Osterwalder V, Guggenheim B. Human root caries: Microbiota of a limited number of root caries lesions. Caries Res, 1996; 30(1): 52-64.

8. van Houte J. Role of micro-organisms in caries etiology. J Dent Res, 1994; 73(3): 672-681.

9. Loesche WJ. Role of Streptococcus mutans in human dental decay. Microbiol Rev, 1986; 50(4): 353-380.

10. Tanzer JM, Livingston J, Thompson AM. The microbiology of primary dental caries in humans. J Dent Educ, 2001; 65(10): 1028-1037.

11. Percival RS, Challacombe SJ, Marsh PD. Age-related microbiological changes in the salivary and plaque microflora of healthy adults. J Med Microbiol, 1991; 35(1): 5-11.

12. Giacaman RA, Araneda E, Padilla C. Association between biofilm-forming isolates of mutans streptococci and caries experience in adults. Arch Oral Biol, 2010; 55(8): 550-554.

13. Schaeken MJ, Creugers TJ, Van der Hoeven JS. Relationship between denta plaque indices and bacteria in dental plaque and those in saliva. $J$ Dent Res, 1987; 66(9): 1499-1502.

14. Kreth J, Merritt J, Shi W, Qi F. Competition and coexistence between Streptococcus mutans and Streptococcus sanguinis in the dental biofilm. J Bacteriol, 2005; 187(21): 7193-7203.

15. Kreth J, Zhang Y, Herzberg MC. Streptococcal antagonism in oral biofilms: Streptococcus sanguinis and Streptococcus gordonii interference with Streptococcus mutans. J Bacteriol, 2008; 190(13): 4632-4640.

16. Loesche WJ, Walenga A, Loos P. Recovery of Streptococcus mutans and Streptococcus sanguis from a dental explorer after clinical examination of single human teeth. Arch Oral Biol, 1973; 18(4): 571-575.

17. Ge Y, Caufield PW, Fisch GS, Li Y. Streptococcus mutans and Streptococcus sanguinis colonization correlated with caries experience in children. Caries Res, 2008; 42(6): 444-448.

18. Caufield PW, Dasanayake AP, Li Y, Pan Y, Hsu J, Hardin JM. Natural history of Streptococcus sanguinis in the oral cavity of infants: Evidence for a discrete window of infectivity. Infect Immun, 2000; 68(7): 4018-4023.

19. van Houte J, Lopman J, Kent R. The final $\mathrm{pH}$ of bacteria comprising the predominant flora on sound and carious human root and enamel surfaces. J Dent Res, 1996; 75(4): 1008-1014.

20. Preza D, Olsen I, Willumsen T, Grinde B, Paster BJ. Diversity and site-specificity of the oral microflora in the elderly. Eur J Clin Microbiol Infect Dis, 2009; 28(9): 1033-1040.

21. Heintze U, Frostell G, Lindgärde F, Trell E. Secretion rate and buffer effect of resting and stimulated whole saliva in relation to general health. Swed Dent J, 1986; 10(5): 213-219.

22. Gold OG, Jordan HV, Van Houte J. A selective medium for Streptococcus mutans. Arch Oral Biol, 1973; 18(11): 1357-1364.
23. Syed SA, Loesche WJ. Survival of human dental plaque flora in various transport media. Appl Microbiol, 1972; 24(4): 638-644.

24. Rogosa M, Mitchell JA, Wiseman RF. A selective medium for the isolation and enumeration of oral lactobacilli. J Dent Res, 1951; 30(5): 682-689.

25. Emilson CG. Prevalence of Streptococcus mutans with different colonial morphologies in human plaque and saliva. Scand J Dent Res, 1983; 91(1): 26-32. 26. Little W, Korts D, Thomson L, Bowen W. Comparative recovery of Streptococcus mutans on ten isolation media. J Clin Microbiol, 1977; 5(6): 578-583.

27. Syed SA, Loesche WJ. Efficiency of various growth media in recovering oral bacterial flora from human dental plaque. App/ Microbiol, 1973; 26(4): 459-465.

28. Llena-Puy $C$. The role of saliva in maintaining oral health and as an aid to diagnosis. Med Oral Patol Oral Cir Bucal, 2006; 11(5): E449-455.

29. Contardo S, Díaz N, Lobos O, Padilla C, Giacaman RA. Oral colonization by Streptococcus mutans and its association with the severity of periodontal disease in adults. Rev Clin Periodoncia Implantol Rehabil Oral, 2011; 4(1): 9-13.

30. van Houte J. Role of micro-organisms in caries etiology. J Dent Res, 1994; 73(3): 672-681.

31. Preza D, Olsen I, Aas JA, Willumsen T, Grinde B, Paster BJ. Bacterial profiles of root caries in elderly patients. J Clin Microbiol, 2008; 46(6): 2015-2021.

32. MINSAL. Diagnostic of the Oral Health Situation. Available at: http://www. redsalud.gov.cl/archivos/salud_bucal/perfilepidemiologico.pdf. Accessed: Feb 2, 2012; 2007.

33. Carlsson J, Grahnen H, Jonsson G, Wikner S. Establishment of Streptococcus sanguis in the mouths of infants. Arch Oral Biol, 1970; 15(12): 1143-1148.

34. van der Hoeven JS, Camp PJ. Mixed continuous cultures of Streptococcus mutans with Streptococcus sanguis or with Streptococcus oralis as a model to study the ecological effects of the lactoperoxidase system. Caries Res, 1993; 27(1): 2630 .

35. De Stoppelaar JD, Van Houte J, Backer Dirks O. The relationship between extracellular polysaccharide-producing streptococci and smooth surface caries in 13-year-old children. Caries Res, 1969; 3(2): 190-199.

36. Loesche WJ, Hockett RN, Syed SA. The predominant cultivable flora of tooth surface plaque removed from institutionalized subjects. Arch Oral Biol, 1972; 17(9): 1311-1325.

37. Pan YP, Li Y, Caufield PW. Phenotypic and genotypic diversity of Streptococcus sanguis in infants. Oral Microbiol Immunol Denmark, 2001; 235-242.

38. Gizani S, Papaioannou W, Haffajee AD, Kavvadia K, Quirynen M, Papagiannoulis $\mathrm{L}$. Distribution of selected cariogenic bacteria in five different intra-oral habitats in young children. Int J Paediatr Dent England, 2009; 193-200.

39. Tong H, Chen W, Merritt J, Qi F, Shi W, Dong X. Streptococcus oligofermentans inhibits Streptococcus mutans through conversion of lactic acid into inhibitory $\mathrm{H} 2 \mathrm{O} 2$ : A possible counteroffensive strategy for interspecies competition. Mol Microbiol England, 2007; 872-880.

40. Loesche WJ. Chemotherapy of dental plaque infections. Oral Sci Rev, 1976; 9: $65-107$

41. Alaki SM, Loesche WJ, da Fonesca MA, Feigal RJ, Welch K. Preventing the transfer of Streptococcus mutans from primary molars to permanent first molars using chlorhexidine. Pediatr Dent, 2002; 24(2): 103-108. 